\title{
Analysis of gamma ray dose for dried up pond storing low enriched $\mathrm{UO}_{2}$ fuel
}

\author{
Yasushi $\mathrm{Nauchi}^{1, *}$, and Motomu Suzuki ${ }^{1}$ \\ ${ }^{1}$ Central Research Institute of Electric Power Industry, Nuclear Risk Research Center, 2-6-1, Nagasaka, Yokosuka, Japan.
}

\begin{abstract}
Gamma ray dose is calculated for loss of coolant accident in spent fuel pond (SFP) storing irradiated fuels used in light water reactors. Influence of modelling of fuel assemblies, source distributions, and loading fraction of fuel assemblies in the fuel rack on the dose are investigated.
\end{abstract}

\section{Introduction}

In systems of light water reactors, discharged spent fuel assemblies (FAs) are stored in racks of a spent fuel ponds (SFP). A FA is stored until its radioactivity decays out below the limit for shipment. A SFP is filled with water which acts as coolant to remove the decay heat and shielding material against intense radiation from the FAs.

By the nuclear disaster occurred at Fukushima DaiIchi (1F) nuclear power station (NPS) on March 11, 2011, risk of loss of coolant accident in SFP (SFP LOCA) has been recognized. In $1 \mathrm{~F}$, the residual heat removal systems (RHR) in unit 1, 2, 3, and 4 were stopped due to the station black out. Even worse, coolant feeding systems were broken by hydrogen explosion in some of the units. Due to larger decay heat, the temperature of the coolant in unit 4 was raised even after the destruction of ceiling of reactor building $(\mathrm{R} / \mathrm{B})$. Therefore, decrement of the coolant level by evaporation was anticipated. In unit 4 of $1 \mathrm{~F}$, its decay heat removal function was recovered by feeding water from a concrete pumping vehicle prepared outside R/B and the SFP was prevented from LOCA, at last [1].

After the accidents, SFP-LOCA becomes one of the design base accidents (DBA) in Japanese NPS [2]. In addition to evaporation following to malfunction of RHR, leakage of coolant by siphon phenomenon initiated by destruction of outlet pipes of SFP cooling and cleanup systems is also lined up on the anticipated sequence to SFP LOCA. In the both cases, utilities have to assess what is happened when active region of FAs is exposed to air. The containment capability of fuel rod would be either intact or not depending on decay heat of FAs, flow of the dry air or vapour, and loading pattern of FAs in racks of SFP. The hazard of internal exposure due to intake of fission products must be estimated only for the case of fuel failure. Contrarily, the hazard of external exposure has to be estimated no matter whether the fuel is intact or not. However, systematic analyses of radiation dose have not been well performed for SFP LOCA.
In this study, we modelled FAs and a SFP and analysed radiation dose at the top height of SFP since the radiation dose at the level would be the source of skyshine radiation. Through the analyses, important parameters to be assessed for the radiation dose were outlined.

\section{Base model and code}

Under the condition where the containment capability of the claddings is intact, all radioactive materials are still remained in the fuel rods. Therefore, radiation transport from source inside the fuel pellets to the top height of the SFP is considered in this work. In this work, a design of PWR 17 x 17 type fuel assembly and a fuel rack was assumed based on published data. Source radiations were calculated for typical burn-up and cooling time. Gamma ray transport was calculated with a continuous energy Monte Carlo code.

\subsection{Calculation geometry and component}

Radiation dose at the top height of the SFP is determined by geometry of FA, the rack, and the SFP. The calculation models of them are described.

\subsubsection{Fuel Assembly}

Actual design information of PWR fuel assemblies are not published except for horizontal array of uranium oxide fuel, cladding, control rod guide tube. Currently, 3 dimensional neutronics calculations have been focused on in the reactor physics field and benchmark models are proposed for the calculations. Among them, BEAVRS benchmark model gives information of intermediate grid spacers, plenums, plenum springs, plugs of rods, and upper and lower nozzles [3]. Since shielding effects of those components are worth to be quantified in this study, the assembly model in the BEAVRS benchmark was

\footnotetext{
*Corresponding author: nauchi@criepi.denken.or.jp
} 
adopted. The components are listed up in table 1 and upper part of the FA is shown in Fig. 1.

Table 1. Modelled components and material in fuel assembly

\begin{tabular}{|l|l|}
\hline component & material \\
\hline fuel & $\mathrm{UO}_{2}$ \\
cladding & Zry-4 \\
end plug & Zry-4 \\
Spacer & Inconel 718 \\
& Zry-4 \\
plenum & Helium \\
plenum spring & Inconel 718 \\
Space & air \\
Lower/upper nozzle & SUS-304 \\
\hline
\end{tabular}

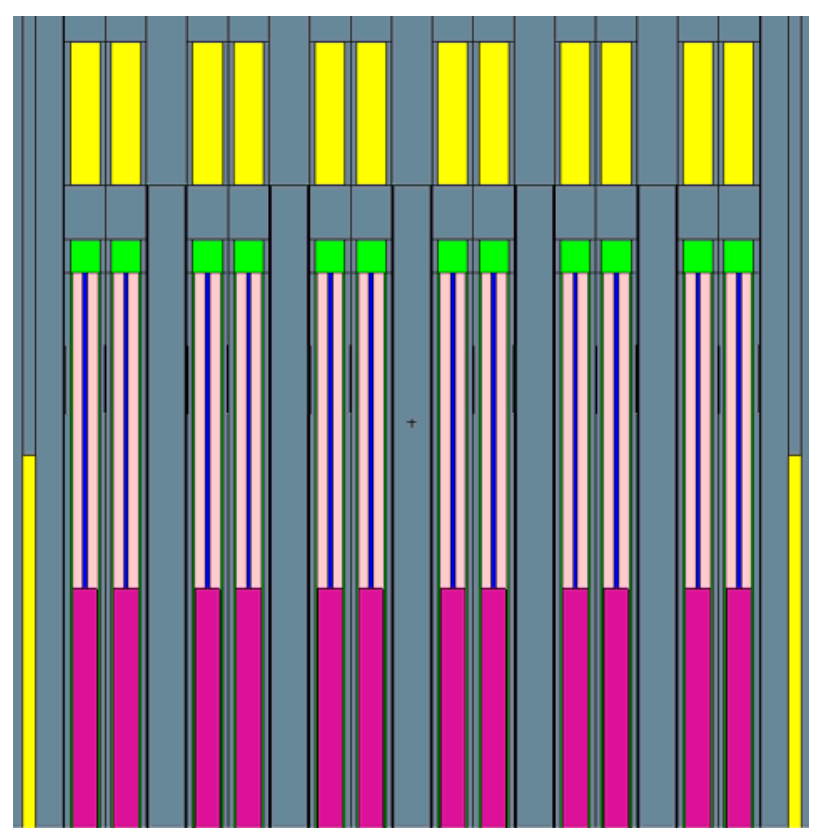

gray: dry air yellow: SUS-304

Green: Zry-4

purple: Inconel-718 magenta: $\mathrm{UO}_{2}$

Fig. 1. Vertical model of top part of fuel assembly.

A fuel rod was modelled by combination of cylinders. A stack of uranium oxide $\left(\mathrm{UO}_{2}\right)$ fuel pellets were modelled by a cylinder rod of the active fuel height $(366 \mathrm{~cm})$, in which dishes or chamfers were smeared. As described later, only the gamma ray dose was considered in this work so that only atomic number densities of uranium and oxygen were assigned in this work. A fuel cylinder rod was enclosed in a rod made of Zircaloy -4 (Zry-4). The plenum spring of spiral shape, actually, was modelled in a co-axial cylinder rod. The space in the rod was filled with helium gas. The both ends of rod were plugged.

In the PWR $17 \times 17$ type FA, 264 fuel rods, 24 control rod guide tubes, and a instrumentation guide tubes are assembled in square shaped array of which fuel rod pitch is $1.26 \mathrm{~cm}$. The pitch is fixed by the grid spacers. There are axially 8 grid spacers in a FA. They were modelled by square pipes. The top and bottom end of the spacers were modelled by Zry-4 and others were by Inconel-718.

The axial position of the spacers are fixed and suspended by control rod guide tubes. The tubes are fixed on upper and lower nozzles. The nozzles were also modelled in array of cylinder columns. Each column was set coaxially with a fuel rod.

\subsubsection{SFP and rack}

The FAs are put into cells of racks fixed on a floor of a SFP. The major role of the rack is to prevent from criticality incidents by keeping a distance between the adjacent assemblies. There are several types of rack employed in Japanese utilities. Among them, the rack made of stainless steel (SS) containing boron (B-SUS) has been popular after the re-racking strategy was taken to enhance capacities of SFPs. In the representative BSUS rack, square pipes were arrayed in square shaped lattice of cell. Although detailed geometries of a rack and a SFP have not been published, we made model geometry as a base case referring to the literature [4].

In the model, the thickness of the wall of the square pipe was $4 \mathrm{~mm}$ and the wide of the pipe was 24 $\mathrm{cm}$. The height of the rack was $4.4 \mathrm{~m}$ and the bottom of the fuel assemblies was assigned $30 \mathrm{~cm}$ above the bottom of the rack. The B-SUS plate was approximated as SUS-304 since contained boron influences little on the gamma ray transport. The horizontal cell pitch was 28 $\mathrm{cm}$. The square pipes located at the center of the cells are separated by spacers equipped at bottom, intermediate, and top of height of the rack, actually. However, such kinds of spacer or joint fittings were assumed as air in the base model. The size of the SFP was set $9 \mathrm{~m} \times 14 \mathrm{~m}$, horizontally, referring to the literature [4]. Considering the cell pitch, the capacity of the rack was determined as $46 \times 27$ FAs. In the base case, all cells are filled with FAs.

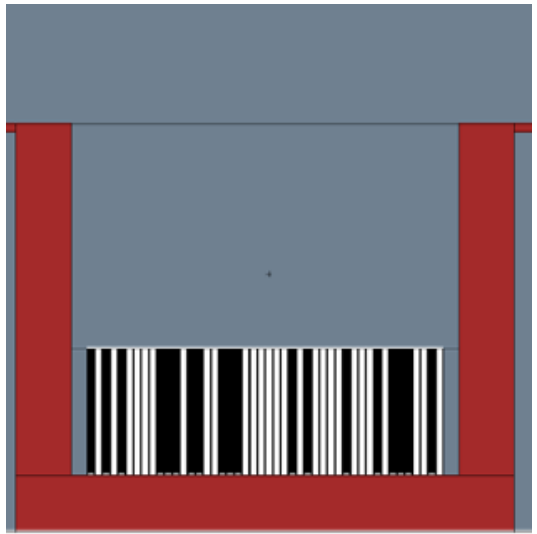

red: concrete, gray: dry air

white: vacant cell, black: cell loaded with fuel

Fig. 2. Vertical model of fuel assembly and spent fuel pond.

The vertical geometry was shown in Fig. 2. The height of the SFP was set $12.4 \mathrm{~m}$. The wall and bottom floor of SFP was concrete of $2 \mathrm{~m}$ in thickness. Metal 
liner on the inner surfaces of the SFP was neglected. The top height of SFP was located on the same level of the surface of the operating floor. The floor was made of concrete and its thickness was $30 \mathrm{~cm}$. In the Base model, the ceiling or the walls of R/B were not modelled and the operating floor was assumed to be extended to the horizontal calculation boundary. The boundary size was $360 \mathrm{~m} \times 360 \mathrm{~m}$ around the center of SFP. Vertically, the lower boundary of the calculation was the bottom of the floor of the SFP and the upper boundary was $2 \mathrm{~km}$ above the operating floor. The SFP and the space above the operating floor were filled with dry air. The temperature of the all materials was assigned $293 \mathrm{~K}$.

\subsection{Source condition}

\subsubsection{Fuel Assembly}

The source strength and its spectrum of gamma rays from FAs were calculated with the ORIGEN-ARP module of the SCALE-6.1 system [5]. In the calculation, the library for PWR 17 x 17 type FA (Westinghouse) was used. No gadolinium rod was considered. Initial ${ }^{235} \mathrm{U}$ enrichment was assigned $4.1 \%$ and the burn-up of the all assemblies were monochromatically determined $48 \mathrm{MWd} / \mathrm{kgHM}$. The cycle length was set 420 days and the power of a FA was assumed constant during the 3 cycles. The cooling time was set 5 years. These kinds of FAs are widely used in Japanese utilities.

Calculated neutron and gamma ray yields were 8.24 $\mathrm{x} 10^{8} / \mathrm{s} / \mathrm{tHM}$ and $1.56 \times 10^{16} / \mathrm{s} / \mathrm{tHM}$, respectively. The former is $7 \sim 8$ orders of magnitude less than the latter. Accordingly, the neutron radiation dose from the spent FA is considered negligibly small. Hereafter, only the gamma ray dose was estimated for radiations by the SFP-LOCA. The gamma ray spectra were estimated in 47 energy groups structure as shown in Fig.3.

\subsubsection{Gamma ray source distribution}

In the present work, the number of gamma ray emission per FA and that per fuel pin were assumed constant over all FAs although burn-up of FAs and fuel pins varies in some extent. Inside the fuel pellet, gamma ray emission points were sampled uniformly.

Contrarily, the axial distribution of gamma ray emission was modelled in detail since the attenuation of gamma rays by $\mathrm{UO}_{2}$ is significant in the estimation of radiation dose. In literature [6], radio activities of ${ }^{134} \mathrm{Cs}$, ${ }^{137} \mathrm{Cs}$ and ${ }^{106} \mathrm{Ru}$ had been measured axially with fine spatial meshes of $2.5 \mathrm{~cm}$ for a spent PWR fuel rod. By averaging the count rates of gamma rays from the three kinds of nuclide, a model of axial distribution of gamma rays was evaluated in this work as shown in Fig. 4. The active length of fuels $(366 \mathrm{~cm})$ in the BEAVRS benchmark is longer than that of gamma ray measured length $(345 \mathrm{~cm})$. In this work, the measured gamma ray distribution was shifted upward so that the top part of the active region of the fuel in the base model radiate gamma rays. Oppositely, the bottom part of $20 \mathrm{~cm}$ would not radiate gamma rays in the base model. The deviation of height of the grid spacers between the geometry model and the gamma ray source distribution one was neglected.

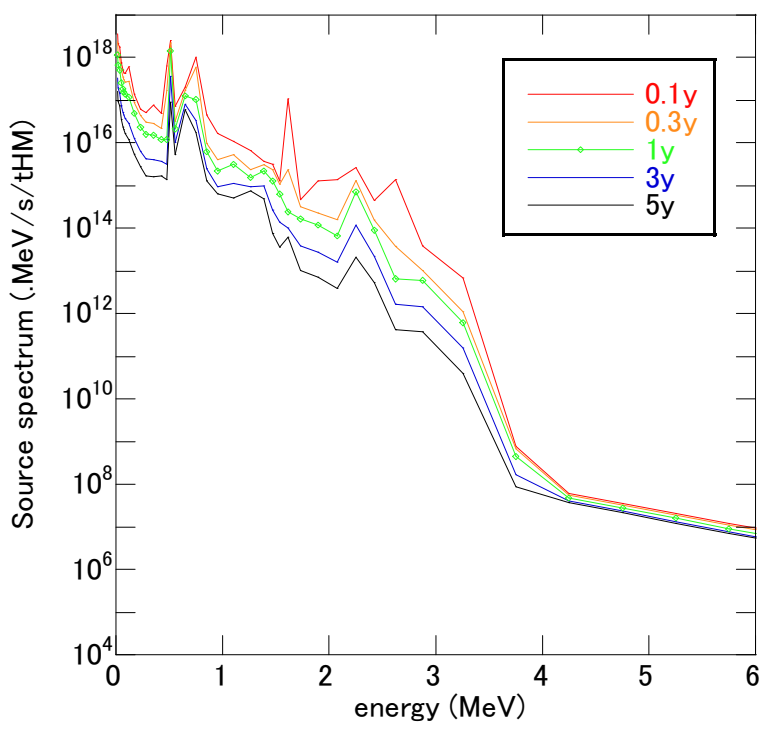

Fig. 3. Gamma ray spectrum from spent fuel in relation to cooling year. Base case is for cooled time of 5years.

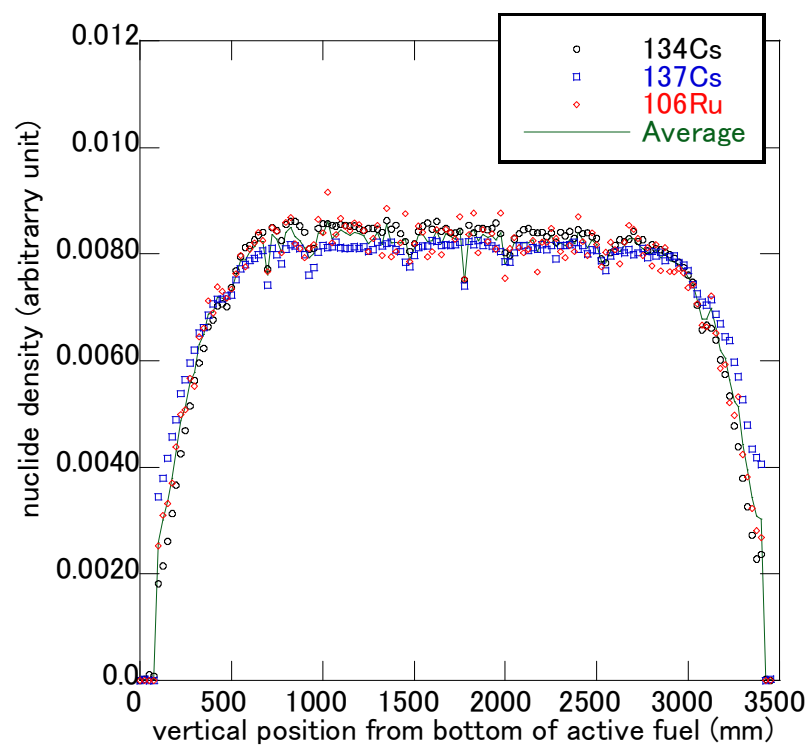

Fig. 4. Axial distribution of gamma ray emission in original literature [6].

\subsection{Gamma ray transport calculation}

Gamma ray transport calculations were performed with the MCNP-5.1.30 code [7]. The coupled transport calculations with either neutron or electron were neglected. The lower energy threshold of the gamma ray transport was set $10 \mathrm{keV}$. Above the energy, the transport was modelled in the "simple mode" where only the photo - electric effect, the Compton scattering, and 
the pair creation processes are considered. The mcplib04 library was used as the cross section [7].

Gamma ray source are assigned as described in section 2.2. The number of source employed was from 100 to 400 milion.

The surface type tally was adopted to calculate average gamma ray flux at the top height of SFP. No bias sampling techniques such as the weight window were applied. The flux to dose conversion factor was referred to ICRP pub 74 [8]. The calculated doses are given per source gamma ray emission. The relative errors of calculated doses were less than $1 \%$ for most of calculations in this work.

\section{Gamma ray dose calculation and results}

In this section, the radiation dose is calculated for the base case. Then, relative to the base case, sensitivities of parameters were studied.

\subsection{Source model}

\subsubsection{Source spectrum}

By MCNP calculation, doses are calculated per source gamma ray. The absolute doses are obtained by multiplying the MCNP results by the source intensity. Varying the coolant time, the source intensity and the spectra were calculated with the ORIGEN-ARP module. The spectra are shown in Fig. 3 together with for the base case which is cooled 5 years. With the spectra, the gamma ray doses were also calculated. The results are written in Table 2.

Table 2. Source intensity and spectrum effect on dose

\begin{tabular}{|r|r|r|r|}
\hline $\begin{array}{r}\text { Cooling } \\
\text { year }\end{array}$ & *dose/source & $\begin{array}{r}\text { *source } \\
\text { intensity }\end{array}$ & $\begin{array}{r}\text { *radiation } \\
\text { dose }\end{array}$ \\
\hline 0.1 & 1.25 & 23.65 & 29.64 \\
0.3 & 0.95 & 12.65 & 12.01 \\
1 & 0.74 & 5.18 & 3.81 \\
3 & 0.90 & 1.81 & 1.63 \\
5 & 1.00 & 1.00 & 1.00 \\
\hline
\end{tabular}

As it is well known, the source intensity declines so rapidly with time during the 1 st year after discharge. Even after a year, the intensity decreases from $520 \%$ to $100 \%$. Therefore, the variation of intensity due to the decay time is essential for the dose estimation. Contrarily, the does per source varies not monotonically with decay time. As shown in Fig.3, the principle emitter of gamma rays varies within a short decay time and the spectrum varies so much. The cooling time effect on the dose per source during a period from 0.1 to 5 years was found about $\pm 25 \%$. The effect is not principle but significant for the precise estimation of the radiation dose.

\subsubsection{Axial distribution of source}

Sensitivity of the number of divisions for the axial distribution of the source was studied here. The sizes considered here were

1) Base case with the fine meshes of $2.5 \mathrm{~cm}$ height

2) 24 coarse meshes of $15.24 \mathrm{~cm}$ height

3) flat distribution along the active height

The distribution of model 2) was estimated by collapsing the fine distribution in model 1). The coarse mesh of the model 2) is widely used for estimating axial burn-up profiles in the reactor physics field. Estimation methods of the gamma ray yield and the spectra are easily to be implemented with a little effort for model 2). The model 3 ) is the easiest way to assume.

The calculated doses for model 2) and 3) are compared to that of model 1) in Table 3 . In model 2), the gamma ray dose is increased by $33 \%$. In model 3 ), radiation dose is done by $145 \%$.

Table 3. Effect of axial distribution model of gamma ray emission on radiation dose

\begin{tabular}{|l|r|}
\hline model & Relative Dose \\
\hline Base Case & 1.000 \\
24 division & 1.328 \\
Flat & 2.446 \\
\hline
\end{tabular}

*data are normalized to base case

In order to study the source distribution effect, further analyses were performed. For the fine meshes applied in model 1), we put gamma ray sources at a certain height and estimated the dose. The calculated value can be regarded as the source importance at the height on the radiation dose. As shown in Fig. 5, the source importance decreases by an order of magnitude only within $15 \mathrm{~cm}$ region from the top of active fuel (TAF). This is due to the fact that $\mathrm{UO}_{2}$ is good shielding material for gamma ray. The slope in Fig. 5 becomes gentle as distance from TAF. In energy region where the pair creation is not dominant, the gamma ray attenuation length is shorter for the lower energy gamma rays. Within a short distance, lower energy gamma rays would be attenuated out. Accordingly, the gamma ray dose by a source at lower height would be dominated by higher energy gamma rays. The gentle slope in the lower region might be due to the attenuation of the higher energy gamma rays.

Due to the attenuation of gammas rays along vertical axis, the meshes of source of $15.24 \mathrm{~cm}$ length is considered still coarse. To take model 3) for the analysis of gamma ray dose during SFP-LOCA introduces extra conservative dose. If they can only take model 2) or 3) for the gamma ray source, some kinds of correction factor should be prepared. 


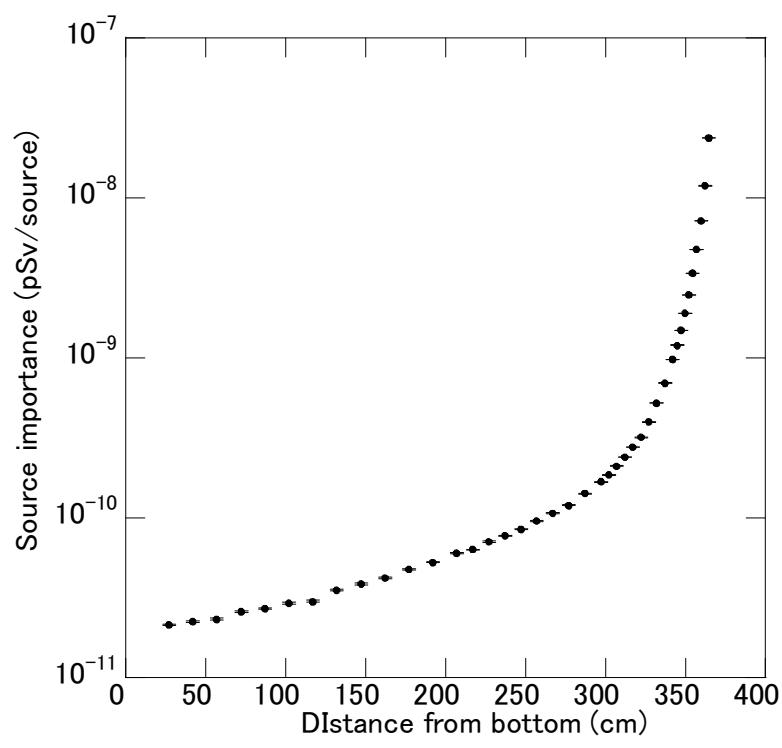

Fig. 5. Axial source importance on radiation dose.

\subsection{Shielding effect of components}

The shielding effect of grid spacers, upper materials above TAF, and walls in the rack cells were calculated for the SFP-LOCA condition. By changing each component in the base case to dry air, variation of the radiation dose was calculated. As listed in Table 4, the upper materials above TAF reduce the dose by $126 \%$ $(2.263 \rightarrow 1)$ since the upper nozzle made of stainless steel, springs made of Inconel-716, upper end plugs and cladding tube made of Zry-4, have significant mass. Also, the effect of the rack cell wall is significant. In order to reduce the extra conservatism, those should be modelled. Contrarily, the effect of the grid spacers is not significant.

Table 4. Shielding effect of component

\begin{tabular}{|l|r|}
\hline shielding material & Relative Dose \\
\hline Base Case & 1.000 \\
Grid spacer & 1.024 \\
materials above TAF & 2.263 \\
rack cell wall & 2.008 \\
\hline
\end{tabular}

*data are normalized to base case

\subsection{Partial loading}

For SFP-LOCA, reduction of the loading fraction of FAs in the rack would be measure to reduce the maximum temperature of claddings to keep integrity of fuel rods. Generally, reduction of the fraction results in decrement of the total gamma ray source in SFP and increment of the source importance. In this section, the influence of the fraction was studied.

\subsubsection{Random loading}

For the rack of $46 \times 27$ cells in the base model, a loading fraction $\xi_{1}$ was uniformly sampled between 0 and 1 . Then for the fraction $\xi_{1},\left(1242 \times \xi_{1}\right)$ cells were filled with FAs and the other $\left(1242 \times\left(1-\xi_{1}\right)\right)$ cells were kept vacant. The filled and vacant cells were also determined randomly as shown in Fig. 6. For tens of sets of random variables, loading patterns of FAs were determined and the radiation doses were calculated. The results are shown in Fig. 7. The gamma ray dose takes a maximal value when the loading fraction is $0.45 \sim 0.5$. Although the number of gamma ray emission is proportional to the fraction, the gamma ray penetration probability increases with the decrement of the fraction. Compared to the base case, the gamma ray dose is increased up to $170 \%$. For the conservative modelling, a kind of optimum search of the loading pattern would be required.

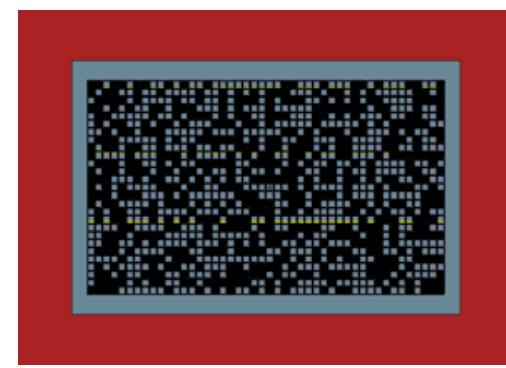

Red: concrete, gray: dry air

Gray in lattice: vacant cell, black: cell loaded with fuel

Fig. 6. Example of random loading of fuel assembly in spent fuel pond

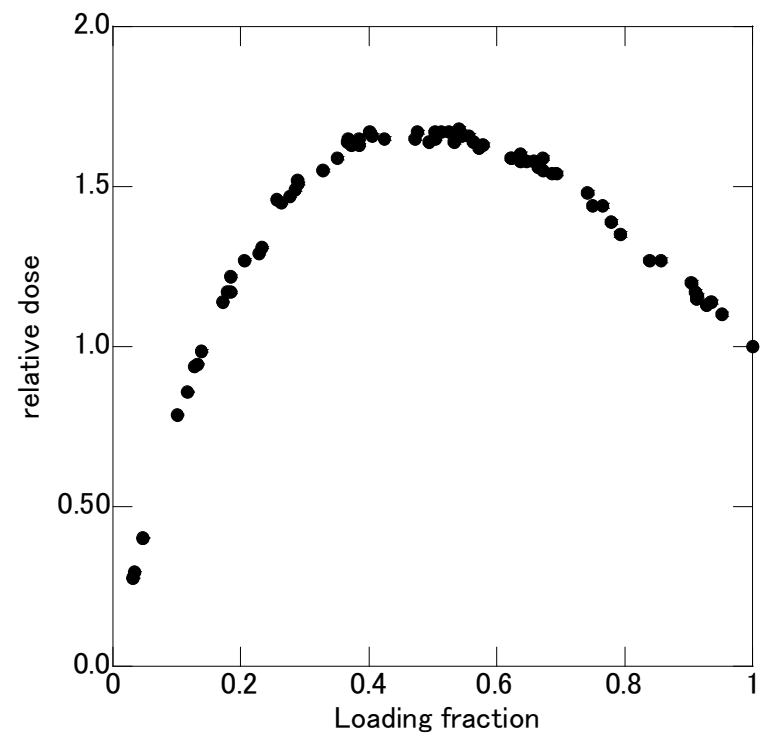

Fig. 7. Gamma ray dose in relation to the loading fraction of fuel assembly in rack. Loading pattern is randomly sampled.

\subsubsection{Systematic loading}

Beginning with the base case for the rack of $46 \mathrm{x}$ 27 cells, other fuel loading patterns were made by discharging or charging FA systematically. 
1) Downsizing the fully charged area as $44 \times 25 \rightarrow 42 \times$ $23 \rightarrow 40 \times 21 \rightarrow \ldots \rightarrow 20 \times 1$.

2) Discharging inner most layer of FAs as shown in Fig. 8 .

Also, additionally two series of loading pattern were considered.

3) Checker board loading of FA by changing checker size.

4) Multi layer of vacant and FA rows.

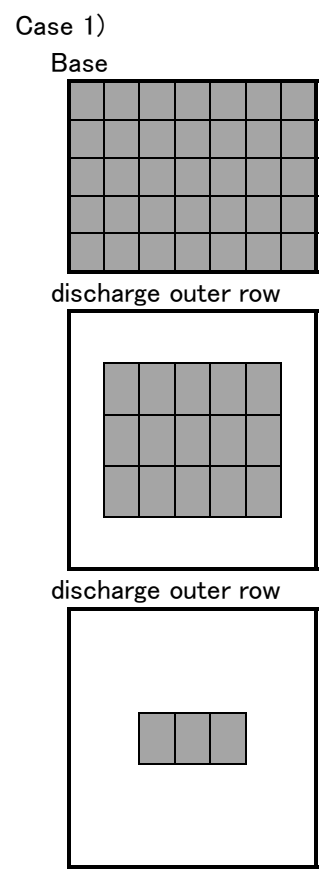

Case 2)

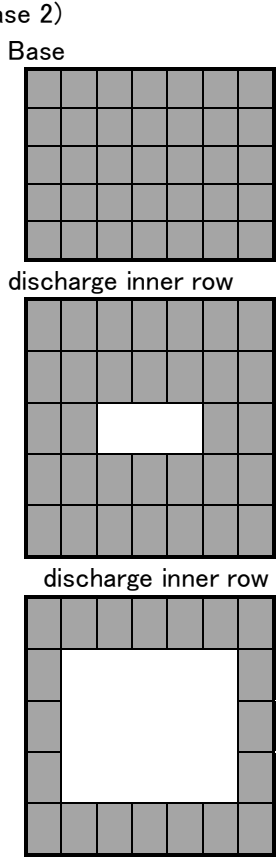

Case 3 checker board

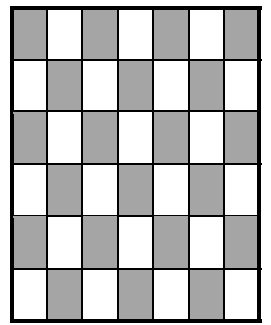

Case4 multi layer
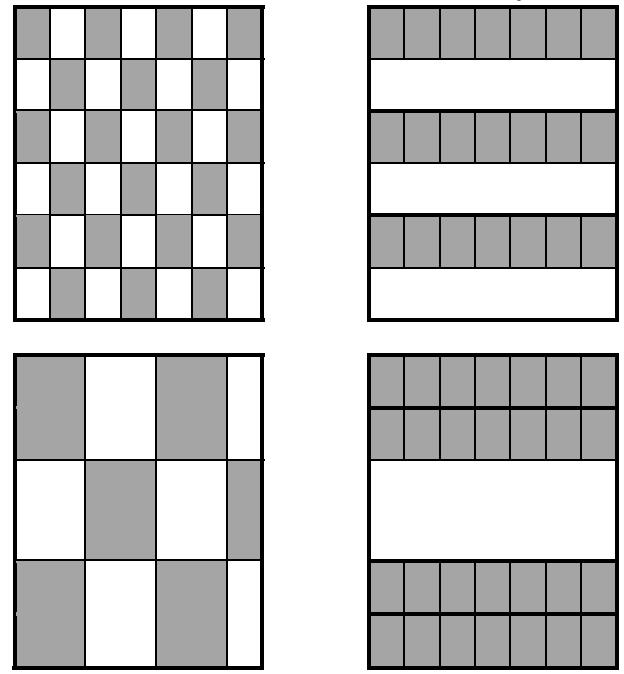

Fig. 8.Fractional loading pattern of fuel assemblies

In Fig. 9, calculate dose and dose per source are shown relative to base case for case 1) and case 2). In both cases, the dose per source increases as FAs are discharged since space is given for gamma ray streaming upward by discharging FAs. However, the increment does not well compensate the decrement of source by discharging. Thus, increment of gamma ray dose like the case of random discharging in Fig. 9 is not observed.
In table 5, calculated gamma ray doses for case 3 ) and 4) are listed. Contrarily to the case 1) and 2), radiation dose increases. Both in case 3) and 4), the gamma ray dose decreases as the size of condensed FAs or the number of rows of FAs are increased. As the size is increased, the number of FA facing 3 or 4 FAs is increased. Such inner FA would not contribute so much to radiation dose at the top height of SFP.

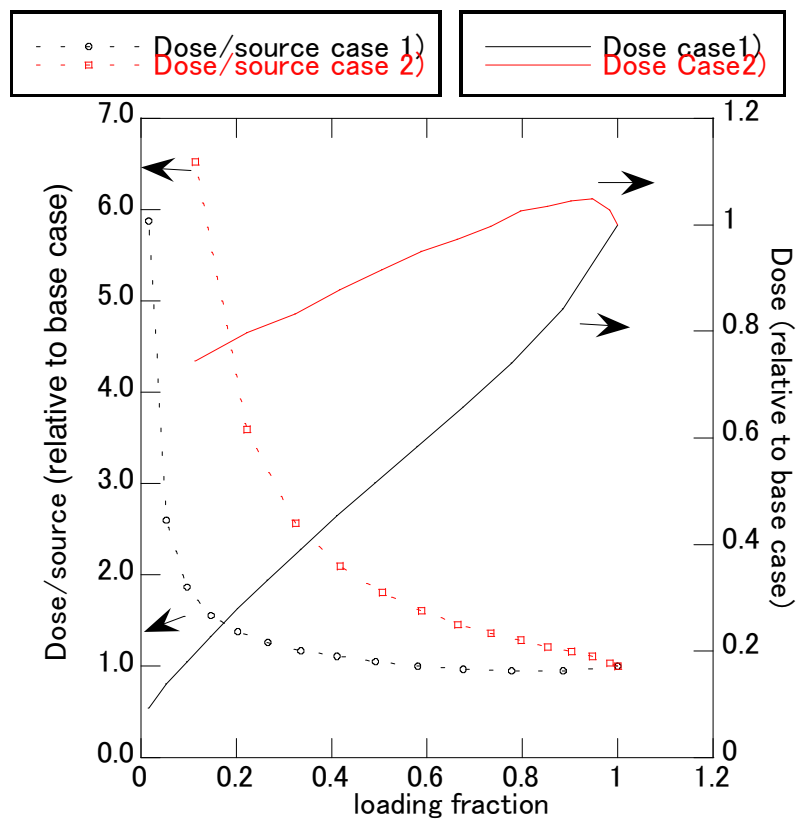

Fig. 9. Gamma ray dose for systematic loading of fuel assembly. Case 1) Downsizing of fuel array. Case 2) Inner most assemblies are discharged From Base case.

Table 5. Relative dose for partially loaded fuel assemblies

\begin{tabular}{|cccc|}
\hline pattern & size & $\begin{array}{c}\text { loading } \\
\text { fraction }\end{array}$ & $\begin{array}{c}\text { relative } \\
\text { dose }\end{array}$ \\
\hline checker & $1 \times 1$ & 0.519 & 1.745 \\
board & $2 \times 2$ & 0.519 & 1.437 \\
& $3 \times 3$ & 0.519 & 1.257 \\
& $4 \times 4$ & 0.556 & 1.185 \\
\hline Multi & 1row & 0.500 & 1.872 \\
Layer & 2row & 0.501 & 1.741 \\
of & 3row & 0.500 & 1.548 \\
rows & 4row & 0.502 & 1.430 \\
\hline
\end{tabular}

*data are normalized to base case

The radiation doses by checker board or multi layer of rows of vacant and FA are greater than the case of random loading pattern. That is greater than base case by $87 \%$ at most. Accordingly, some kind of periodic patterns should be studied further to find conservative loading pattern of FAs. 


\subsection{Rack pitch}

From a view point of the criticality safety, the rack pitch should be wider when the boron concentration in the square can is decreased. The pitch length and the concentration are chosen taking the cost and the benefit into account. Thus, the cell pitch of a rack varies with NPS. Here, the pitch length effect was studied.

Within the size of SFP in the current model, the cell pitch can be increased up to $30 \mathrm{~cm}$ for $46 \times 27$ cells. Here the case was called wide case. Oppositely, a narrow case was also made by assigning the pitch $26 \mathrm{~cm}$. We calculate the relative dose for the case the rack cells are fully filled with the FAs. As shown in Table 6, the radiation dose increases from 0.77 to 1.31 by the increment of the pitch by $15 \%$. For the variation of cell pitch, the effect of axial source modelling studied in section 3.1.2 was studied. As listed in Table 7, the effect is mitigated for wider cell pitch. By using wider pitch, gamma rays from source at lower height in a FA can stream upward between adjacent FAs. Accordingly, the radiation dose at the top height of SFP would be insensitive to the model of the axial distribution of gamma ray source.

Table 6. Pitch length of rack cell and radiation dose

\begin{tabular}{|l|rrr|}
\hline cell pitch $(\mathrm{cm})$ & 26 & 28 & 30 \\
\hline radiation dose & 0.766 & 1.000 & 1.308 \\
\hline
\end{tabular}

Table 7. Effect of axial modelling of source on dose in relation to pitch length of rack cell

\begin{tabular}{|l|rrr|}
\hline cell pitch $(\mathrm{cm})$ & 26 & 28 & 30 \\
\hline fine case & 1.000 & 1.000 & 1.000 \\
24 division & 1.354 & 1.328 & 1.275 \\
Flat & 2.478 & 2.446 & 2.300 \\
\hline
\end{tabular}

*data are normalized to fine case for each cell pitch

\section{Summary and conclusion}

Focusing on SFP-LOCA, calculation models for radiation dose at the top height of SFP were surveyed in the case where all radioactive materials are remained in fuel rods. A FA, rack, and SFP are modelled referring published data such as specifications of BEAVRS benchmark model. The dose calculation consists of evaluations of source and radiation transport.

It has been well known that cooling time of fuel assembly is essential for the radiation dose since it is major parameter to determine the source intensity. In this study, spectrum effect of source was additionally identified as $\pm 25 \%$.

One of the major findings here is the strong shielding effect of FA itself. Even to use axial distribution of gamma ray source in 24 divisions was found still coarse and overestimate the radiation dose by $33 \%$. Conventional flat source model would give so conservative dose. The conservatism is comparative to neglect shielding effect of the wall of rack cell and components of FA equipped above the top of active fuel. The effect of the latter was found thanks to the BEAVRS benchmark model.

Significance of loading pattern in FAs was also identified. By reducing loading fraction by $\sim 50 \%$, the gamma ray dose would be increased by $87 \%$ although source intensity is reduced by the $\sim 50 \%$. That is due to enhancing gamma ray streaming upward by the discharging half of the FAs.

Generally, measures to mitigate SFP-LOCA take costs. Therefore, the authors consider the reasonable assessment of risk of radiation hazard is desirable. In the assessment, assumptions to cause extra conservatisms should be taken care of referring this work.

\section{References}

1. Tokyo Electric Power Company, "Fukushima Nuclear Accident Analysis Report (Interim Report)"HP http://www.tepco.co.jp/en/press/corpcom/release/betu11 e/images/111202e14.pdf, browse on Sep. 02,2016

2. https://www.nsr.go.jp/data/000034411.pdf,in Japanese, on internet, browse on Sep. 02,2016, for example.

3. N. Horelik, B. Herman, B. Forget, and K. Smith, "Benchmark for Evaluation and Validation of Reactor Simulations (BEAVRS)", Proceedings of International Conference on Mathematics and Computational Methods Applied to Nuclear Science and Engineering (M\&C 2013), Sun Valley, Idaho, p.2986-2999, May 5-9 (2013).

4. Kansai Electric Power Company, press release, http://www.kepco.co.jp/corporate/pr/2004/0409-

1 2j.html, in Japanese, on internet, browse on Aug. $31,2016$.

5. I. C. Gauld, G. Radulescu, G. Ilas, et al., "Isotopic depletion and decay methods and analysis capabilities in SCALE," Nuclear Technology volume 174 p. 169 , May 2011.

6. A. Sasahara, et al., "Neutron and gamma ray source evaluation of LWR high burn-up UO2 and MOX spent fuels," Journal of Nuclear Science and Technology, vol. 41 (4), p.448-456, (2004).

7. X-5 Monte Carlo Team, MCNP - A General Monte Carlo N-Particle Transport Code, Version 5, LAUR-03-1987 (2003).

8. "Conversion Coefficients for Radiological Protection Quantities for External Radiation Exposures," ICRP PUBLICATION 74. 